\title{
Gastric tumours in FAP
}

\author{
Sarah-Jane Walton ${ }^{1,2}$ - Ian M. Frayling ${ }^{3}$ - Susan K. Clark ${ }^{1,2}$ - Andrew Latchford ${ }^{1,2}$
}

Published online: 7 March 2017

(C) Springer Science+Business Media Dordrecht 2017

\begin{abstract}
Gastric cancer is not a recognised extra-colonic manifestation of FAP, except in countries with a high prevalence of gastric cancer. Data regarding gastric adenomas in FAP are sparse. The aim of this study was to review the clinical characteristics of gastric tumours occurring within an FAP population from the largest European polyposis registry. All patients that developed a gastric adenoma or carcinoma were identified from a prospectively maintained registry database. The primary outcome measure was the occurrence of gastric adenoma or adenocarcinoma. Secondary outcomes included APC mutation, tumour stage, management and survival. Eight patients developed gastric cancer and 21 an adenoma (median age 52 and 44 years, respectively). Regular oesophagogastroduodenoscopy surveillance was performed in 6/8 patients who developed cancer. Half were advanced T3/4 tumours and 6/8 had nodal or metastatic spread at diagnosis. All cancer cases died within a median of 13.5 months from diagnosis. Gastric adenomas were evenly distributed: $11 / 21(52 \%)$ in the distal and 10/21 (48\%) proximal stomach, whereas 5/8 (63\%) cancers were located proximally. An association between gastric tumour and desmoid development was observed; 7/8 (88\%) cancer and 11/21 (52\%) adenoma cases had a personal or family history of desmoid. It would appear from this small, retrospective study that gastric cancer is not a prominent
\end{abstract}

Sarah-Jane Walton

sarah-janewalton@nhs.net

1 The Polyposis Registry, St Mark's Hospital, Watford Road, Harrow, London, UK

2 Department of Surgery and Cancer, Imperial College London, Harrow, London, UK

3 Institute of Cancer and Genetics, Cardiff University, Harrow, London, UK extra-colonic feature of FAP in the Western world. It seems to present at an advanced stage with a poor prognosis. There may be an association between gastric tumour and desmoid occurrence but a large multicentre cohort is necessary to investigate this further.

Keywords Gastric cancer - Gastric adenoma - Desmoid · Familial adenomatous polyposis

\section{Introduction}

Familial adenomatous polyposis (FAP) is a rare autosomal dominantly inherited condition due to a germline mutation in the $A P C$ gene. It is a cancer predisposing syndrome that typically results in the development of hundreds to thousands of colorectal adenomas that inevitably progress to cancer by the age of 40 years without prophylactic colectomy or proctocolectomy [1]. Although the colorectal manifestations of FAP predominate, the condition is also associated with the development of several other tumours. Duodenal cancer and desmoid tumours are the leading cause of morbidity and mortality in this population once the colorectal cancer risk has been addressed $[2,3]$.

Gastric cancer is not a prominent extra-colonic manifestation of FAP in the Western world. The risk of developing gastric cancer in this patient group is reportedly similar to that of the general population and less than 1\% [4-6]. However, in Korea and Japan, where gastric cancer is more prevalent, it does seem to be associated with FAP, and the risk is seven to tenfold of that seen in the respective general populations [7-10]. The risk of gastric adenoma development in patients with FAP shows a similar increase in Asian populations compared to the Western world. Similarly, the risk of gastric adenoma development in patients 
with FAP is lower in the Western world compared to Asian populations. In the West, gastric adenomas are reported to occur in $2-10 \%$ of patients [11-13] but ranges between $15-50 \%$ in Asian populations [14-17].

Studies that describe the clinical features of gastric lesions occurring in association with FAP in Western populations are lacking. One such study reported upper GI lesions in FAP and detected gastric dysplasia in only 6 of 102 patients studied endoscopically, with a predilection for the antrum [18]. The largest study in the Western world to report gastric adenoma occurrence involved only 9 cases [13]. The aim of this descriptive study was to report the clinical features of gastric adenomas and carcinomas arising in patients with FAP attending our institution.

\section{Methods}

All histologically confirmed gastric adenocarcinoma cases were identified from the polyposis registry database. Upper GI tract endoscopic surveillance was introduced in 1974 and gastric adenomas discovered between 1974 and 2015 were identified from the same database. Medical notes, imaging, endoscopy and pathology reports were obtained and further scrutinised. Data gathered included patient demographics, APC mutation, tumour location, histology and stage, intervention undertaken and survival outcome. Gastric lesions were classified as proximal or distal, relative to the gastric incisura.

Permission was obtained to access and utilise data from an international FAP database held by the International Society for Gastrointestinal Hereditary Tumours (InSiGHT) group. This database provided a large FAP cohort that could be used to identify further gastric cancer cases and provide a spectrum and frequency of mutations in FAP overall to compare with our gastric adenoma and cancer group. This database was larger and less likely to be biased. Gastric cancer cases obtained from this database were only used for $A P C$ mutation evaluation and excluded from other clinical analyses as these data were largely unavailable from the database. Entries with ambiguous terminology (for example 'atypical FAP') were excluded from the study. In FAP approximately $95 \%$ of germline $A P C$ mutations are frameshift or nonsense mutations, resulting in a truncated protein with an abnormal function [19]. Large APC mutations that would not allow genetic analysis when searching for a potential mutation cluster region for gastric cancer were excluded, as were all silent and missense mutations. Only those mutations that would predict truncation of the APC protein (non-sense, frameshift and splicing mutations) were included. The site of the $A P C$ germline mutation determines that of the 'second hit' mutation, resulting in an optimal level of beta-catenin activity [20]. These 'first hit-second hit' associations can influence phenotype, with germline mutations $3^{\prime}$ of codon 1400 resulting in more upper gastrointestinal lesions and desmoid tumours [21, 22].

Gastric adenoma cases were not obtained from the InSiGHT database as these were not routinely reported and detailed pathology findings were unavailable.

$A P C$ mutations in the gastric cancer group were analysed to determine the spectrum of sites involved. If a gastric cancer genotype/phenotype correlation exists, it would most likely occur within this area of the gene. The proportion of $A P C$ mutations in the control group lying within and outside of this region was then recorded and compared to the gastric adenoma and cancer groups.

\section{Statistical analysis}

Statistical analysis was performed using GraphPad Prism 6 (GraphPad, San Diego, CA). For analysis a p-value of less than 0.05 was considered significant.

\section{Results}

A search of the polyposis registry database revealed eight cases of gastric cancer and 21 patients with gastric adenomas. An exact denominator is not possible to define. However, 1435 patients were registered who have had all, or at least part of their care at our institution. The majority of the patients were of white European ethnicity with a minority originating from the Indian subcontinent.

\section{Clinical features of gastric adenocarcinomas}

Eight British Caucasian patients (five female) known to the polyposis registry developed gastric cancer (Table 1). The median age at diagnosis was 52 years (range 41-64). Two patients were asymptomatic and diagnosed at the time of their surveillance OGD. Half of patients presented with anaemia, one of whom had recurrent bleeding through a jejunostomy. Abdominal pain was a presenting feature in only two cases.

Despite 6/8 patients undergoing regular upper GI endoscopic surveillance for their FAP, half of these patients presented with advanced tumours, stage T3 or T4. All of these advanced lesions, along with a $\mathrm{T} 2$ tumour, were associated with metastatic disease at the time of diagnosis. All patients had extensive cystic gland polyps carpeting the stomach but no gastric adenomas were reported from histology obtained at endoscopy. Most tumours were located in the proximal stomach (fundus $n=1$, cardia $n=1$ and body $n=3$ ) with only two located in the antrum. The two most proximal tumours were $\mathrm{T} 3$ and $\mathrm{T} 4$ stage, while 
Table 1 Clinical data comparing gastric adenoma and gastric adenocarcinoma cases in patients with FAP

\begin{tabular}{|c|c|c|c|}
\hline & $\begin{array}{l}\text { Gastric } \\
\text { adenomas } \\
\mathrm{n}=21(\%)\end{array}$ & $\begin{array}{l}\text { Gastric cancers } n=9 \\
(\%)\end{array}$ & $\mathrm{p}$ value \\
\hline \multicolumn{4}{|l|}{ Gender } \\
\hline Male & $11(52)$ & $3(38)$ & $0.70 *$ \\
\hline Female & $10(48)$ & $5(62)$ & \\
\hline $\begin{array}{l}\text { Median age at } \\
\text { diagnosis, years } \\
\text { (range) }\end{array}$ & $44(23-71)$ & $52(41-64)$ & $0.06 * *$ \\
\hline \multicolumn{4}{|l|}{ Location and T-stage } \\
\hline Cardia & $1(5)$ & $1(13) \mathrm{T} 3$ & \\
\hline Fundus & $5(24)$ & $1(13) \mathrm{T} 4$ & $0.48^{+}$ \\
\hline Body & $4(19)$ & $3(37) \mathrm{T} 2$ & \\
\hline Antrum & $11(52)$ & $2(25) \mathrm{T} 1, \mathrm{~T} 3$ & \\
\hline Unknown & & $1(13) \mathrm{T} 4$ & \\
\hline \multicolumn{4}{|l|}{ Spigelman stage } \\
\hline $0-\mathrm{II}$ & $11(52)$ & 0 & \\
\hline III & $7(33)$ & $1(13)$ & $0.0007^{+}$ \\
\hline IV & $3(14)$ & $6(75)$ & \\
\hline Unknown & & $1(13)$ & \\
\hline \multicolumn{4}{|c|}{ Desmoid tumour history } \\
\hline Personal & $9(43)$ & $5(62)$ & \\
\hline Family & $2(9)$ & $2(25)$ & $0.14^{*}$ \\
\hline Neither & $10(48)$ & $1(13)$ & \\
\hline \multicolumn{4}{|c|}{$A P C$ mutation distribution } \\
\hline Codon range & $233-1461$ & $685-2040$ & \\
\hline $3^{\prime}$ of codon 1390 & $2(10)$ & $6(67)$ & $0.005^{*}$ \\
\hline $5^{\prime}$ of codon 1390 & $17(81)$ & $3(33)$ & \\
\hline Unknown & 1 & & \\
\hline Excluded & 1 & & \\
\hline
\end{tabular}

*Fisher's exact test, **Mann Whitney-U test, ${ }^{+} \chi^{2}$ test

the three tumours located in the stomach body were all $\mathrm{T} 2$. Distally one tumour was only T1 and the other T3.

Treatment for gastric cancer comprised chemotherapy alone for half of the patients, who all had metastatic and/or nodal disease. Surgery was performed in three cases: a partial and total gastrectomy for the T1 and T2 tumours without nodal or metastatic disease and a sleeve gastrectomy for a T3 tumour with metastatic disease that was diagnosed at the time of the surgery to stem ongoing blood loss (previous biopsies had all been benign). One patient with a T4 tumour and widespread metastatic disease received supportive care for their gastric tumour.

Median survival from diagnosis was 13.5 months (range 2-101) and all patients eventually died as a direct result of their gastric cancer.

Seven out of eight (88\%) patients had significant duodenal polyposis (Spigelman stage III-IV disease), although information was unavailable for the remaining patient.
Seven patients $(88 \%)$ had a personal history or family history of a desmoid tumour.

\section{Gastric adenoma cases}

Twenty-one (11 male) patients with gastric adenoma were identified from the registry database and all were of British Caucasian ethnicity. The median age at detection was 44-years (range 23-71), eight years younger than that for gastric adenocarcinoma (Table 1). Histology found 18 $(86 \%)$ of lesions were tubular adenomas (TA) and three were tubulovillous adenomas (TVA). Eighteen (86\%) had low grade dysplastic lesions and three (14\%) had highgrade dysplasia; all of the lesions which contained high grade dysplasia were detected in the proximal stomach. Adenomas were found almost equally in the proximal $(\mathrm{n}=10,48 \%)$ and distal stomach $(\mathrm{n}=11,52 \%)$. Proximal lesions were usually a pale, creamy coloured flat plaque, whereas the antral lesions were more pinkish and elevated. All gastric adenomas larger than $2 \mathrm{~cm}$ were located in the proximal stomach. In eight out of 21 (38\%) cases multiple adenomas were identified. In $7 / 8$ of these cases the adenomas were located within the same region but for one case an adenoma was found in both the fundus and antrum.

Patients who developed gastric cancer had more advanced duodenal polyposis (Spigelman stage III or IV disease) compared to the adenoma group, $7 / 8(88 \%)$ and $10 / 21$ (47\%), respectively (Logrank test $\chi^{2}=14.63$ (2df), $\mathrm{p}=0.0007)$. However, this may be explained by the age discrepancy between the two cohorts. Indeed, in the adenoma group the median age of those with Spigelman stage III or IV duodenal disease was 49 years compared to 40 years for those with stage 0 -II disease.

Ten (47\%) of the gastric adenoma cohort had Spigelman III or IV disease. A personal or family history of desmoid disease was observed in 11/21 (52\%).

To date no patients have required surgery for gastric adenomas. One patient has been lost to follow up. All other patients have been managed endoscopically and have either had $(9 / 21(43 \%))$ or are awaiting endoscopic resection of their gastric adenoma.

\section{$A P C$ mutation analysis}

Further analysis of the APC mutation distribution was undertaken comparing patients with gastric cancers and gastric adenomas with controls with FAP but no reported gastric pathology obtained from the InSiGHT database. A total of 3012 patients were identified from the InSiGHT database and were of predominantly European ethnicity, of which one had gastric cancer and 38 were recorded as having possible gastric adenoma. These 38 patients were not included in the adenoma group analysis as detailed 
histology reports were not available to confirm these findings. The adenoma cases from the polyposis registry database were compared with the remaining 2974 FAP control cases from the InSiGHT database. For one gastric adenoma case the $A P C$ mutation was not known and in another case was a large deletion of Exon 1-5. This was excluded from $A P C$ mutations analysis as this would null the gene rather than produce a truncating protein and could not be used to compare potential phenotypes.

All gastric cancer cases had germline mutation between codons 685 and 2040, whereas only $64 \%$ of controls cases had a mutation found within this region (Table 2; Fig. 1). Only 10/21 (48\%) of patients with a gastric adenoma had an $A P C$ mutation in this region (Table 3). Gastric cancer cases were significantly more likely to have a mutation $3^{\prime}$ of codon 1390 compared to controls, 6/9 (67\%) and 399/3012 $(13 \%)(\mathrm{p}=0.0003)$, respectively; this is close to the region associated with a higher risk for desmoid development [23]. Germline mutation in adenoma cases had a similar distribution to that in controls (2/21 (10\%) 3' of codon 1390).

Table 2 Comparing frequency of $A P C$ mutations within and outside of the region for gastric cancer mutations with FAP controls

\begin{tabular}{llll}
\hline APC mutation (codon) & Controls, $\mathrm{n}(\%)$ & $\begin{array}{l}\text { Gastric cancer } \\
\text { cases, n }(\%)\end{array}$ & $\mathrm{p}$ value \\
\hline 5' of 685/3' of 2040 $^{\prime}$ & $1107(37)$ & 0 & $0.03^{*}$ \\
Between 685 and 2040 & $1905(63)$ & $9(100)$ & \\
$5^{\prime}$ of codon 1390 & $2613(87)$ & $3(33)$ & $0.0003^{*}$ \\
$3^{\prime}$ of codon 1390 & $399(13)$ & $6(67)$ & \\
\hline
\end{tabular}

*Fisher's exact test
Table 3 Comparing frequency of $A P C$ mutations within and outside of the region for gastric cancer mutations between FAP controls and gastric adenoma cases

\begin{tabular}{llll}
\hline$A P C$ mutation (codon) & Controls, n (\%) & Gastric adenomas & $\mathrm{p}$ value \\
\hline $5^{\prime}$ of 685/3' of 2040 & $1096(37)$ & $9(43)$ & $0.35^{\mathrm{a}}$ \\
Between 685 and 2040 & $1878(63)$ & $10(48)$ & \\
Unknown & & 1 & \\
Excluded & & 1 & $1^{\mathrm{a}}$ \\
5'of codon 1390 & $2576(87)$ & $17(81)$ & \\
3'of codon 1390 & $398(13)$ & $2(10)$ & \\
Unknown & & 1 & \\
Excluded & & 1 & \\
\hline
\end{tabular}

${ }^{\mathrm{a}}$ Fisher's exact test

\section{Discussion}

This study reports the clinical features and outcomes from a rare cohort of patients with FAP and gastric cancer within a predominantly European population. There are no other descriptive studies of gastric cancer in patients with FAP in the Western world. The only study to report the clinical characteristics of gastric cancers occurring in FAP originates from a Japanese population with distinct differences from our data [10]. This study reported on five patients and found three had multifocal disease whereas in our series all eight had a single focus of tumour. We also reported that more were located in the proximal stomach whereas there was no clear distribution for the Japanese tumours. In addition, our study found most gastric cancers presented late

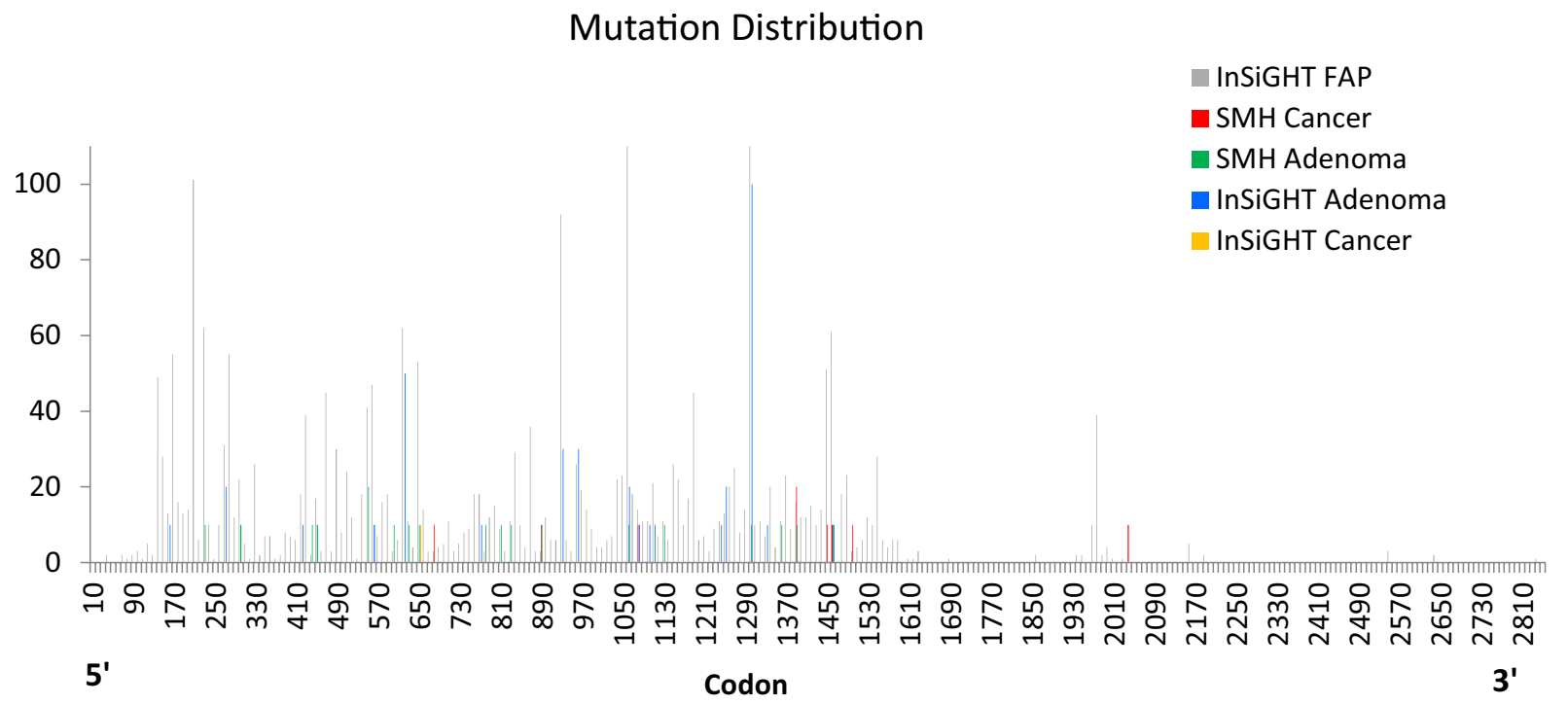

Fig. 1 Graph demonstrating the distribution of APC 5' to $3^{\prime}$ mutations, InSiGHT and SMH (St Mark's Hospital) FAP, gastric cancer and adenoma databases 
in their course whereas in the Japanese group they were detected at an earlier stage.

This was a small series but it supports previous study findings that gastric cancer is not a significant extra-colonic manifestation of FAP in the West $[4,5]$. In contrast, Park et al. found the incidence of gastric adenomas was $14 \%$ and gastric cancers nearly $3 \%$ in Korean patients with FAP [14].

We found gastric cancer occurred at a relatively young age (median age of 52 years), presented with subtle signs or without any symptoms at all, and had a dismal prognosis. Three-quarters of the patients in our cohort presented with advanced tumours at diagnosis, despite the majority undergoing endoscopic surveillance. This may indicate a significant gastric adenoma and carcinoma miss rate. It is important to note that extensive carpeting by cystic gland polyps occurred in these cases and this may have hindered prompt gastric cancer diagnosis by obscuring the presence of tumour. However, most tumours were located in the body and fundus, including two of the three advanced T3/4 tumours. In our experience, adenomas in this region have a subtle pale plaque-like appearance that can be more difficult to detect endoscopically than the sessile pink polypoid lesions that were usually found in the antrum (Figs. 2, 3), further impeding the pick-up rate for proximal lesions. Ngamruengphong et al. reported a significant adenoma miss rate at endoscopy affecting 6/9 patients [13]. A recent systematic review and metaanalysis reported an endoscopic miss rate for the detection of gastric cancers in a non-FAP population as high as $10 \%$, particularly when lesions were located in the

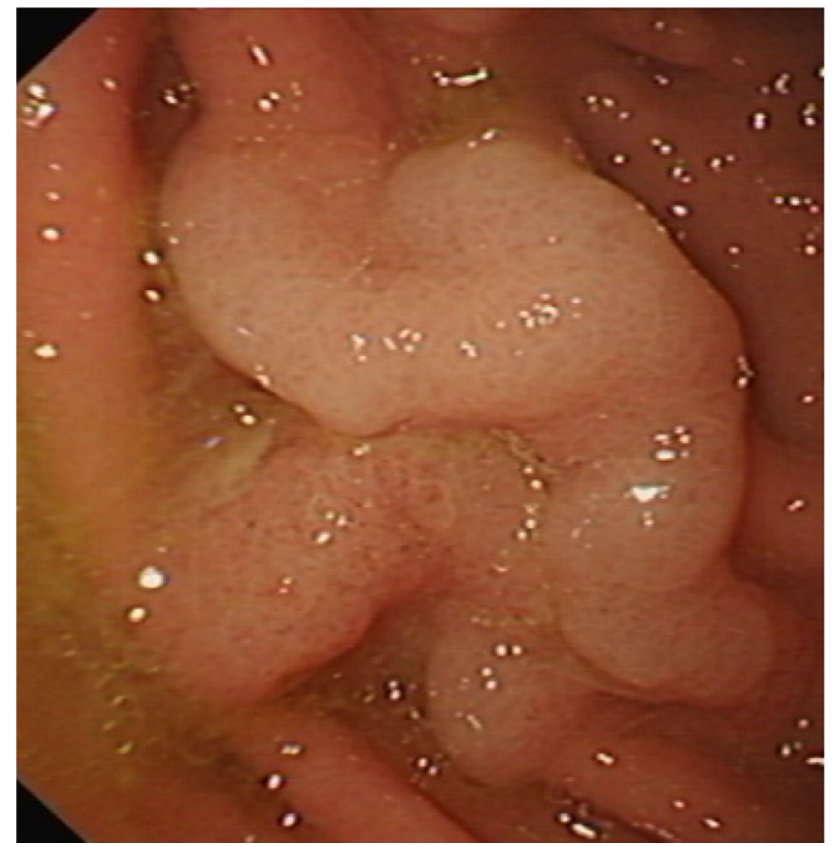

Fig. 2 A subtle plaque-like gastric adenomas at the fundus

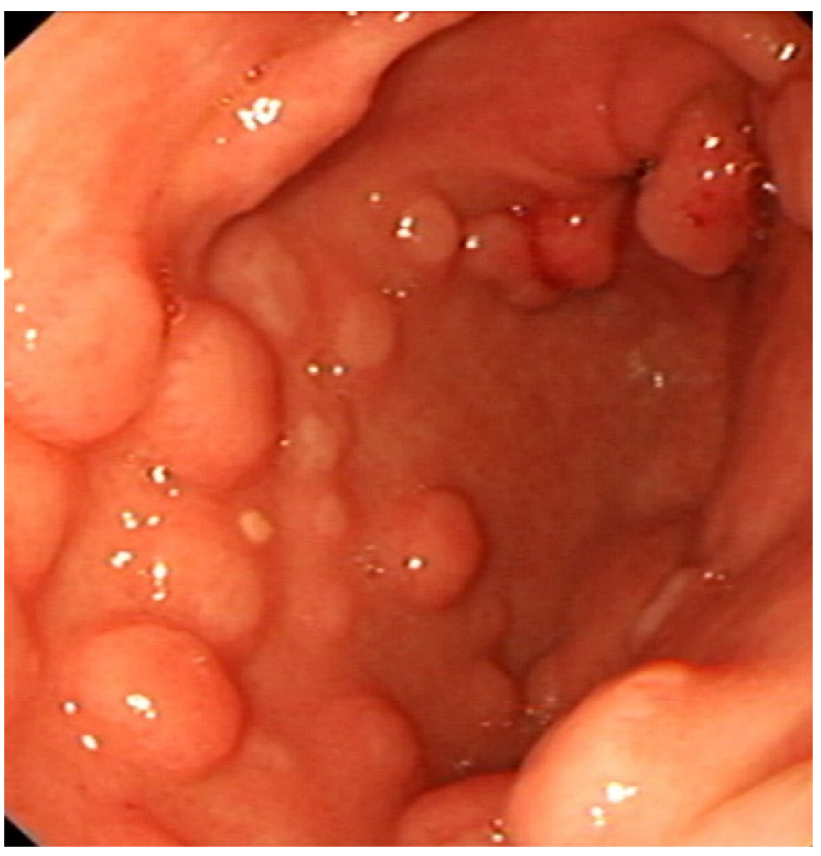

Fig. 3 Polypoid antral adenomas

body [24]. Advances in endoscopic equipment, training and techniques are likely to have improved our ability to detect subtle plaque-like lesions that may have been missed previously. This is supported by the findings from our study that the majority of gastric adenomas (15/21) were detected in recent years (between 2013 and 2015). Because of these issues it is impossible to quantify the prevalence of gastric adenomas in patients with FAP. It is also difficult to extrapolate their clinical course. It is likely that they have a relatively benign clinical course, that may be analogous to the duodenal adenoma, however, the occurrence of three adenomas with high grade dysplasia in this study highlights the malignant potential of these lesions.

A significant difference was noted between the cancer group and adenoma group regarding the presence of advanced duodenal disease (Spigelman III/IV). Advanced duodenal disease was seen more commonly in the cancer group (88\%) than the adenoma group $(47 \%)$. The two groups however are not age matched (the median age of adenoma group was eight years younger than the cancer group) and therefore it is difficult to comment further on whether or not there is an association between gastric cancer and duodenal disease in FAP. To explore further a possible association between gastric adenomas and/or cancer with duodenal disease would require a large, age matched control group for comparison. Spigelman et al. previously reported that patients with gastric adenomas had a higher incidence of severe duodenal polyposis [18]. A more recent study by Ngamruengphong et al. failed to show an 
association between gastric adenomas and duodenal disease in FAP [13].

An interesting finding from this study was the possible association between gastric cancer development and a history of desmoid tumour. Sixty-two per cent of these patients had a desmoid tumour, a significantly higher rate than typically reported in patients with FAP (up to $25 \%$ ) $[23,25](p=0.036)$, Fisher's exact test. If such an association were real it could suggest a genotype-phenotype correlation for gastric cancer occurrence in FAP exists, in the same way as for desmoid tumours [26]. They may share a common region or overlap at a particular point on the $A P C$ gene (for example $3^{\prime}$ of codon 1399 for desmoids and 1390 for gastric cancer). Significantly more germline mutations were identified $3^{\prime}$ of codon 1390 in the gastric cancer group compared to controls. Another explanation could be that both groups share a common modifier gene.

It would seem logical that gastric adenomas, (if they are the pre-malignant lesion responsible for gastric cancer development in FAP), would demonstrate similar findings. Indeed, a greater proportion of gastric adenoma cases than would normally be expected had a personal or family history of desmoid. Interestingly, Ngamruengphong et al. also reported that patients with FAP and a gastric adenoma were significantly more likely to have had a desmoid tumour compared to patients without a history of a gastric adenoma, 5/9 (56\%) and 19/88 (22\%), respectively [13]. This was comparable to our study's findings. Case reports have also described an association between gastric cancer and desmoid tumour development following gastrectomy [27, 28].

The germline $A P C$ mutation distribution in gastric cancer cases differs from that seen in gastric adenomas, which more closely reflects the control FAP group. The mutation clustering seen in gastric cancer cases may therefore be an anomalous finding due to the small numbers of patients in this cohort. The observation of an association despite the differences in germline mutation distribution could also indicate an association between gastric neoplasia and desmoid disease which is independent of the germline mutation.

The retrospective nature of this study is a limitation, despite the data being collected prospectively. In a few instances, patients with FAP will undergo upper GI surveillance locally, rather than the tertiary centre. This might lead to fewer adenoma cases being identified through the registry database then would normally occur if undertaken at the tertiary centre. Despite this, most patients undergo their routine FAP follow-up at this institution and therefore OGD reports from local institutions are usually obtained by the polyposis registry. Certainly, the development of a gastric cancer is unlikely to occur without the tertiary centre being aware. The InSiGHT database contained a large
FAP cohort but it was not possible to include those patients labelled as 'gastric adenoma' in the adenoma group analysis as there were insufficient data regarding histology details to be sure they were true gastric adenoma cases. Referral bias is unlikely to have occurred as the tertiary hospital is not an upper GI cancer centre and therefore patients would not have been referred specifically for the purpose of managing their gastric adenoma or cancer.

The strengths of this study include the polyposis registry database that has existed for over 90 years, providing one of the largest cohorts of patients with FAP for the study of this rare disease. Access to the InSiGHT database also provided thousands of FAP 'control' cases without gastric lesions. This was an invaluable resource for studying $A P C$ mutations in this group.

\section{Conclusion}

This is the largest series to report on gastric adenomas and gastric cancers occurring in association with FAP in the Western World. Unlike Far East populations, gastric cancer is not a feature of FAP and when diagnosed they are often advanced, despite OGD surveillance, and associated with a poor prognosis. There may be an association between the development of gastric adenomas or gastric cancers and desmoid tumours in FAP. Although the existence of a gastric-cancer genotype-phenotype correlation seemed a possibility, this was not supported in the adenoma group. To determine the true significance of these findings a much larger cohort is necessary, requiring a considerable international, multi-centre, collaborative effort.

Acknowledgements We would like to thank Stefan Aretz, curator of the InSiGHT database, for providing access to the database.

\section{References}

1. Bisgaard ML, Fenger K, Bulow S, Niebuhr E, Mohr J (1994) Familial adenomatous polyposis (FAP): frequency, penetrance, and mutation rate. Hum Mutat 3:121-125

2. Arvanitis ML, Jagelman DG, Fazio VW, Lavery IC, McGannon E (1990) Mortality in patients with familial adenomatous polyposis. Dis Colon Rectum 33:639-642

3. Bertario L, Presciuttini S, Sala P, Rossetti C, Pietroiusti M (1994) Causes of death and postsurgical survival in familial adenomatous polyposis: results from the Italian Registry. Italian Registry of Familial Polyposis Writing Committee. Semin Surg Oncol 10:225-234

4. Offerhaus GJ, Giardiello FM, Krush AJ, Booker SV, Tersmette AC, Kelley NC, Hamilton SR (1992) The risk of upper gastrointestinal cancer in familial adenomatous polyposis. Gastroenterology 102:1980-1982

5. Garrean S, Hering J, Saied A, Jani J, Espat NJ (2008) Gastric Adenocarcinoma Arising from Fundic Gland Polyps in a Patient 
with Familial Adenomatous Polyposis Syndrome. Am Surg 74:79-83

6. Wood LD, Salaria SN, Cruise MW, Giardiello FM, Montgomery EA (2014) Upper GI tract lesions in familial adenomatous polyposis (FAP): enrichment of pyloric gland adenomas and other gastric and duodenal neoplasms. Am J Surg Pathol 38:389-393

7. Park JG, Park KJ, Ahn YO, Song IS, Choi KW, Moon HY, Choo SY, Kim JP (1992) Risk of gastric cancer among Korean familial adenomatous polyposis patients. Report of three cases. Dis Colon Rectum 35:996-998

8. Iwama T, Mishima Y, Utsunomiya J (1993) The impact of familial adenomatous polyposis on the tumorigenesis and mortality at the several organs. Its rational treatment. Ann Surg 217:101-108

9. Utsunomiya J, Miki Y, Kuroki T, Furuyama J (1988) Recent trends in studies on carcinogenesis in familial adenomatous polyposis. Gan To Kagaku Ryoho 15:185-191

10. Shibata C, Ogawa H, Miura K, Naitoh T, Yamauchi J, Unno M (2013) Clinical characteristics of gastric cancer in patients with familial adenomatous polyposis. Tohoku J Exp Med 229:143-146

11. Sarre RG, Frost AG, Jagelman DG, Petras RE, Sivak MV, McGannon E (1987) Gastric and duodenal polyps in familial adenomatous polyposis: a prospective study of the nature and prevalence of upper gastrointestinal polyps. Gut 28:306-314

12. Bianchi LK, Burke CA, Bennett AE, Lopez R, Hasson H, Church JM (2008) Fundic gland polyp dysplasia is common in familial adenomatous polyposis. Clin Gastroenterol Hepatol 6:180-185

13. Ngamruengphong S, Boardman LA, Heigh RI, Krishna M, Roberts ME, Riegert-Johnson DL (2014). Gastric adenomas in familial adenomatous polyposis are common, but subtle, and have a benign course. Hered Cancer Clin Pract 12:4

14. Park SY, Ryu JK, Park JH, Yoon H, Kim JY, Yoon YB, Park JG, Lee SH, Kang SB, Park JW et al (2011) Prevalence of gastric and duodenal polyps and risk factors for duodenal neoplasm in korean patients with familial adenomatous polyposis. Gut Liver 5:46-51

15. Iida $\mathrm{M}$, Yao $\mathrm{T}$, Itoh $\mathrm{H}$, Watanabe $\mathrm{H}$, Matsui $\mathrm{T}$, Iwashita $\mathrm{A}$, Fujishima M (1988) Natural history of gastric adenomas in patients with familial adenomatosis coli/Gardner's syndrome. Cancer 61:605-611

16. Nakamura S, Matsumoto T, Kobori Y, Iida M (2002) Impact of Helicobacter pylori infection and mucosal atrophy on gastric lesions in patients with familial adenomatous polyposis. Gut 51:485-489

17. Yamaguchi $T$, Ishida $H$, Ueno $H$, Kobayashi $H$, Hinoi $T$, Inoue $Y$, Ishida F, Kanemitsu Y, Konishi T, Tomita N et al (2016) Upper gastrointestinal tumours in Japanese familial adenomatous polyposis patients. Jpn J Clin Oncol 46:310-315
18. Spigelman AD, Williams CB, Talbot IC, Domizio P, Phillips RK (1989) Upper gastrointestinal cancer in patients with familial adenomatous polyposis. The Lancet 2:783-785

19. Fearnhead NS, Britton MP, Bodmer WF (2001) The ABC of APC. Hum Mol Genet 10:721-733

20. Lamlum H, Ilyas M, Rowan A, Clark S, Johnson V, Bell J, Frayling I, Efstathiou J, Pack K, Payne S et al (1999) The type of somatic mutation at APC in familial adenomatous polyposis is determined by the site of the germline mutation: a new facet to Knudson's 'two-hit' hypothesis. Nat Med 5:1071-1075

21. Crabtree M, Sieber OM, Lipton L, Hodgson SV, Lamlum H, Thomas HJ, Neale K, Phillips RK, Heinimann K, Tomlinson IP (2003) Refining the relation between 'first hits' and 'second hits' at the APC locus: the 'loose fit' model and evidence for differences in somatic mutation spectra among patients. Oncogene 22:4257-4265

22. Groves C, Lamlum H, Crabtree M, Williamson J, Taylor C, Bass S, Cuthbert-Heavens D, Hodgson S, Phillips R, Tomlinson I (2002) Mutation cluster region, association between germline and somatic mutations and genotype-phenotype correlation in upper gastrointestinal familial adenomatous polyposis. Am J Pathol 160:2055-2061

23. Sinha A, Tekkis PP, Gibbons DC, Phillips RK, Clark SK (2011) Risk factors predicting desmoid occurrence in patients with familial adenomatous polyposis: a meta-analysis. Colorectal Dis 13:1222-1229

24. Pimenta-Melo AR, Monteiro-Soares M, Libanio D, DinisRibeiro M (2016). Missing rate for gastric cancer during upper gastrointestinal endoscopy: a systematic review and meta-analysis. Eur J Gastroenterol Hepatol

25. Vasen HF, Moslein G, Alonso A, Aretz S, Bernstein I, Bertario L, Blanco I, Bulow S, Burn J, Capella G et al (2008) Guidelines for the clinical management of familial adenomatous polyposis (FAP). Gut 57:704-713

26. Church J, Xhaja X, LaGuardia L, O'Malley M, Burke C, Kalady M (2015) Desmoids and genotype in familial adenomatous polyposis. Dis Colon Rectum 58:444-448

27. Komatsu S, Ichikawa D, Kurioka H, Koide K, Ueshima Y, Shioaki Y, Lee CJ, Mutoh F, Hosokawa Y, Oka T et al (2006) Intraabdominal desmoid tumor mimicking lymph node recurrence after gastrectomy for gastric cancer. J Gastroenterol Hepatol 21:1224-1226

28. Kim PS, Han DS, Han HS, Bang HY (2014) Intra-abdominal fibromatosis after gastrectomy for gastric cancer. Ann Surg Treat Res 87:331-335 